\title{
Improvements in monitoring and biochemical control of hypothyroidism in primary care with the use of an electronic protocol: 12-month follow up evaluation
}

\section{Tran AD1,2, Hyer SL2, Rodin DA2, Johri N3, Hickey J4, Dayan CM5, Okosieme OE5}

1. Shadbolt Park House Surgery, Worcester Park, UK. 2. Department of Endocrinology, Epsom and St Helier University Hospitals NHS Trust, Carshalton, UK.

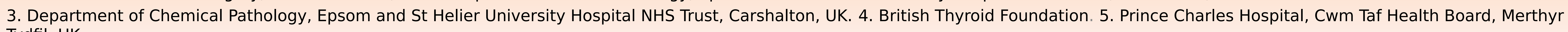
Tydfil, UK

\section{Introduction}

Thyroid hormone replacement is frequently suboptimal. The causes are multi-factoria and there are to date no proven interventions to improve management in primary care.

Following the introduction of the Quality Outcome Framework (QOF), 98-100\% patients with treated primary hypothyroidism received annual TSH checks during the period 2009-20141,2. However, there was no evidence annual monitoring by itself resulted in improved care ${ }^{3,4}$.

In 2017, we developed an electronic Protocol for Monitoring Patients on Thyroxine in General Practice (e-Prompt GP), an electronic alert system in EMIS web designed to 1) emulate the former QOF thyroid e-alerts to test annual thyroid function, and 2) to address out of range thyroid function tests in patients with primary hypothyroidism treated with levothyroxine. We re-audited after 12 months to assess impact. This study presents the results of the 12 -month re-audit.

\section{Aim}

To investigate the impact of an electronic protocol on the monitoring and management of levothyroxine replacement in patients treated for primary hypothyroidism in primary care.

\section{Methods}

Five Surrey GP practices using EMIS web with a total population of 74,984 patients participated in the study.

'e-Prompt GP' Protocol was installed at participating practices.

We ran searches using EMIS Population Reporting manager at baseline and twelve months after the introduction of the electronic protocol.

We analysed the percentage of patients who had:

1) TSH checked in the preceding 12 months

2) Latest TSH level within the local laboratory reference range (0.35-5.0mU/l)

3) TSH both within the local laboratory reference range and checked in last 12 months

\section{E-PROMPT GP PROTOCOL ALERTS ALERTS:}

1. Annual thyroid function test in all patients coded with hypothyroidism, on levothyroxine, subclinical hypothyroidism either coded or based on latest TFT results, previous radio- iodine treatment or currently on amiodarone or lithium 2. Last TSH outside local reference range in patients on levothyroxine

3. Currently pregnant women on levothyroxine or coded with hypothyroidism with latest TSH $>3$

4. Patients on levothyroxine and coded hypothyroid but had not been issued levothyroxine in last 6 months

\section{Exclusions:}

- Children

- Low TSH and coded with secondary hypothyroidism, panhypopituitarism or thyroid cancer

- Patients currently on antithyroid therapy and levothyroxine (block and replace)

\section{Examples of alerts}
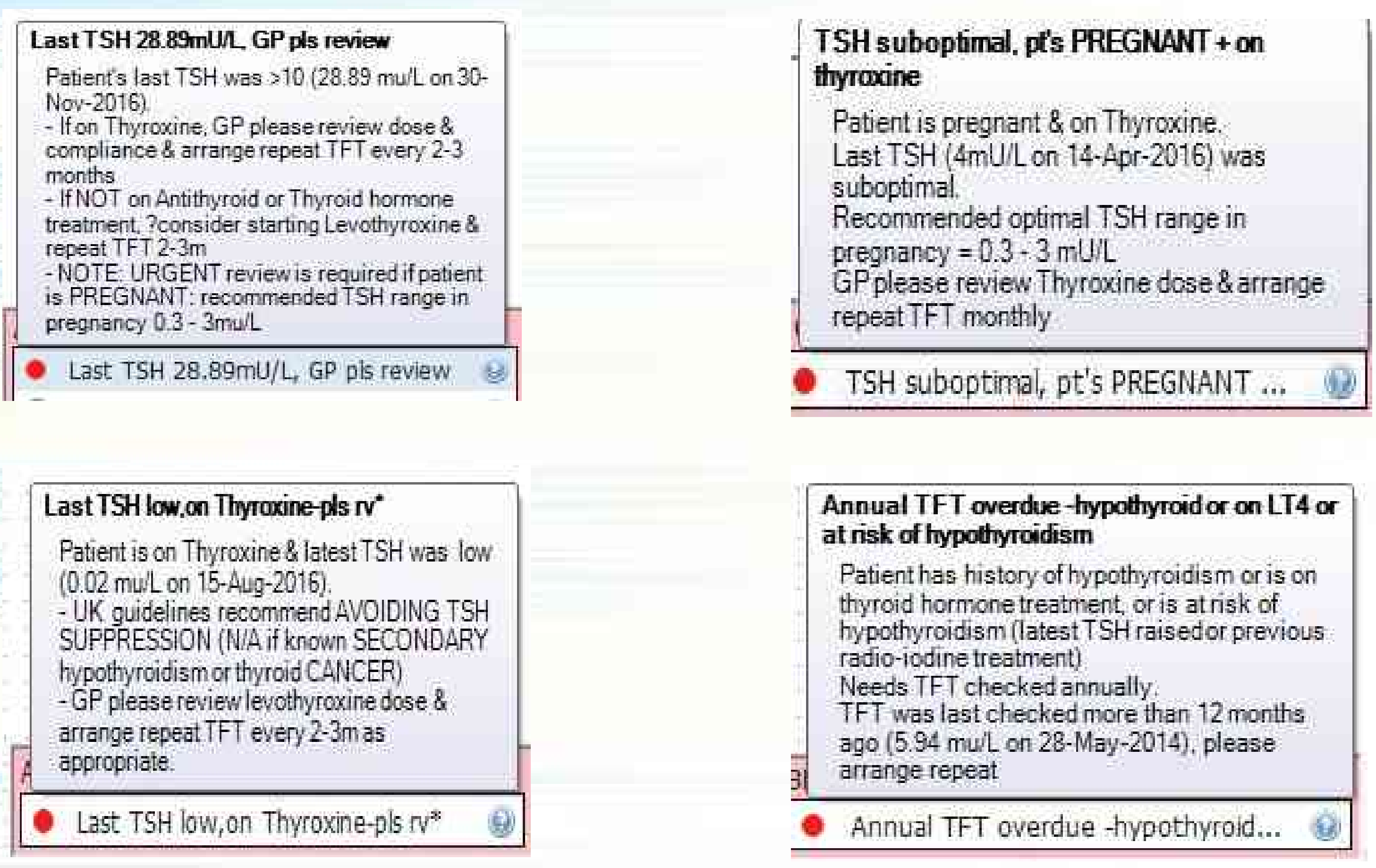

\section{Results}

- The prevalence of treated primary hypothyroidism was $3.2 \%$ and did not change significantly over the course of the study.

- The proportion of patients with TSH checked in the previous 12 months increased from $77 \%$ to $83 \%$.

- The latest TSH result (any date) was within local reference range in $68 \%$ at baseline and improved to $71 \%$ twelve months following introduction of the electronic thyroid e-alerts.

- The proportion of patients with TSH both within local reference range and checked in last 12 months improved from $53 \%$ at baseline to $58 \%$ twelve months after protocol installation.

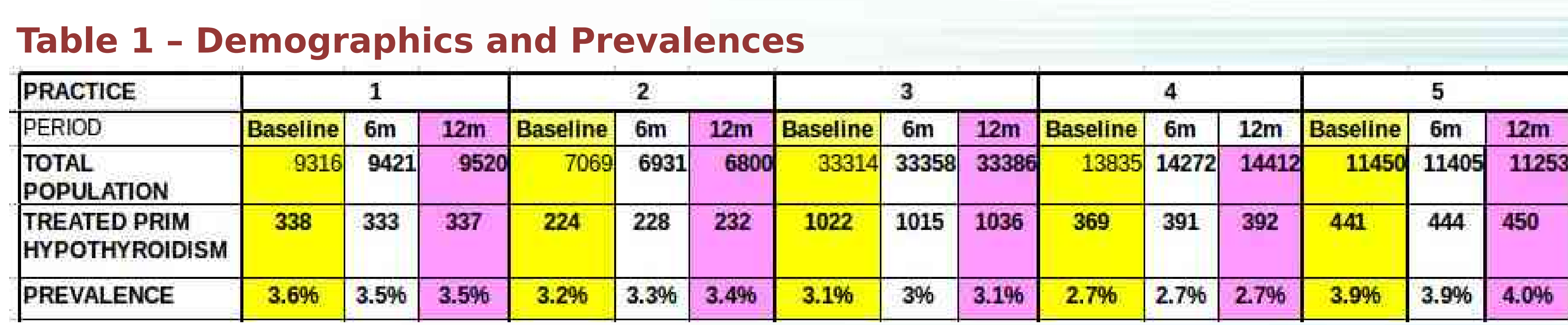

Figure 1 - TSH checked in last 12 months

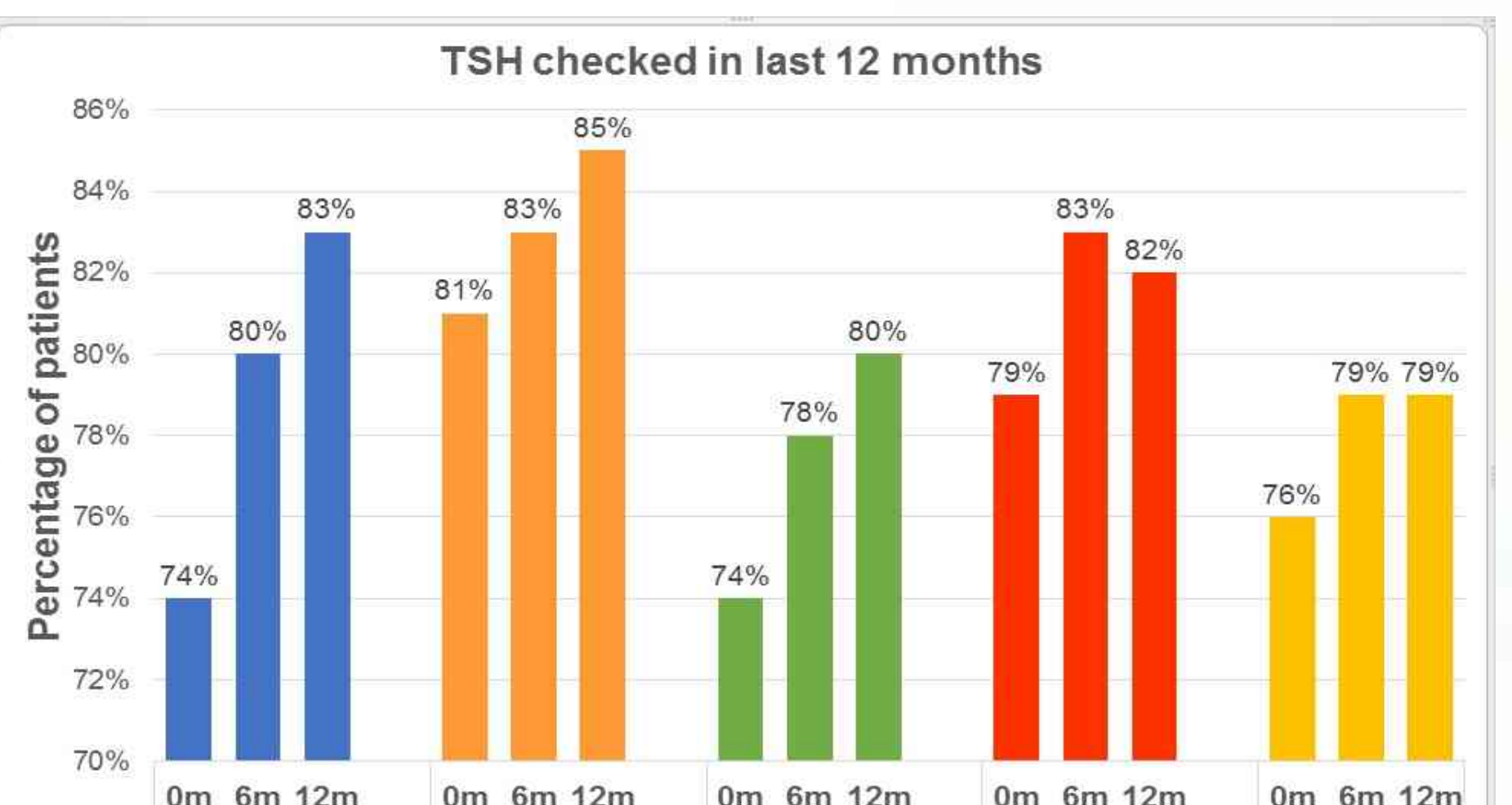

Figure 2 - Last TSH within local reference range

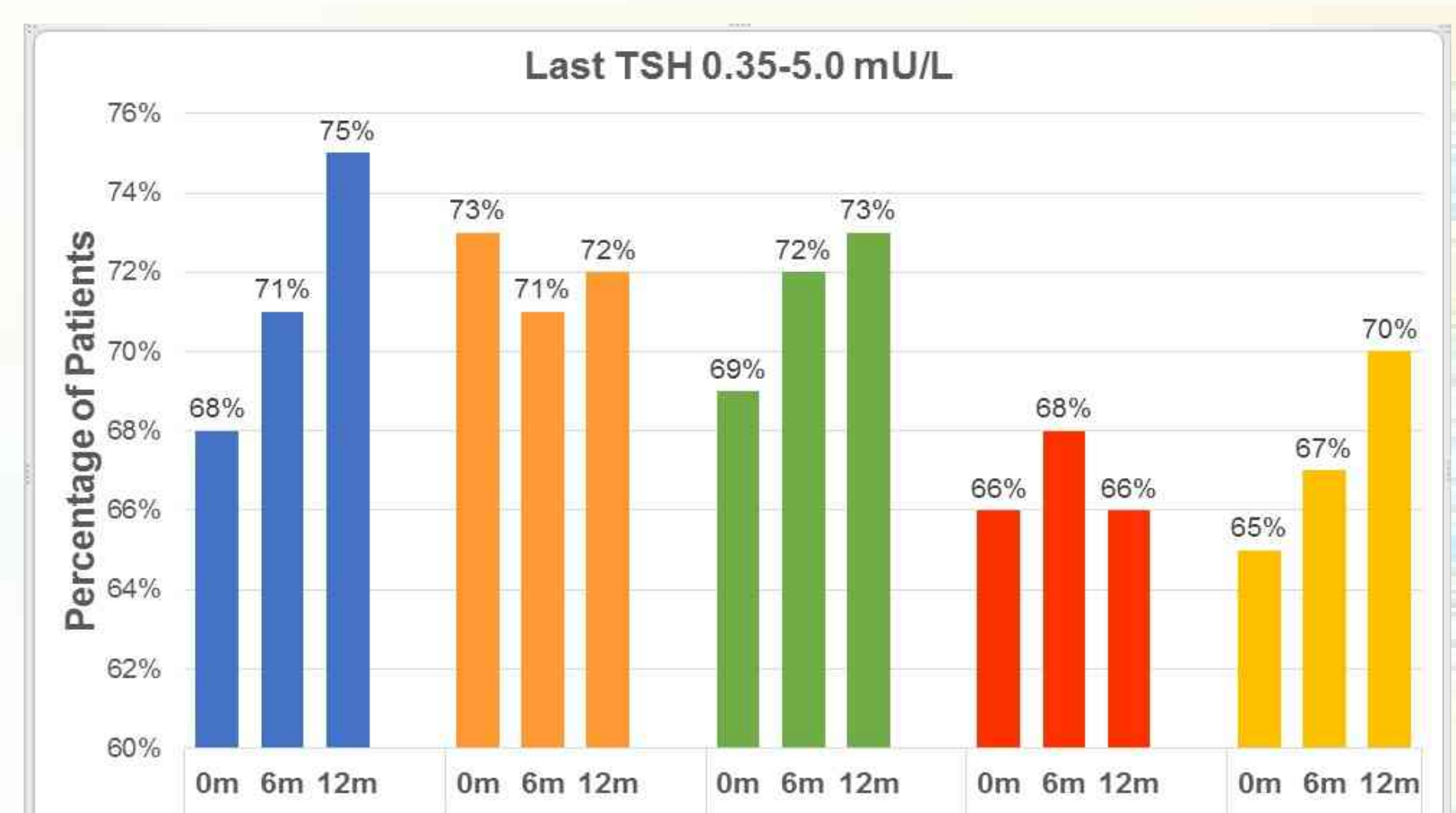

Note: Numbers and prevalence of patients with hypothyroidism at baseline is denoted below the bars for each practice in tables 2

Figure 3 - Last TSH within local reference range and checked in last 12 months

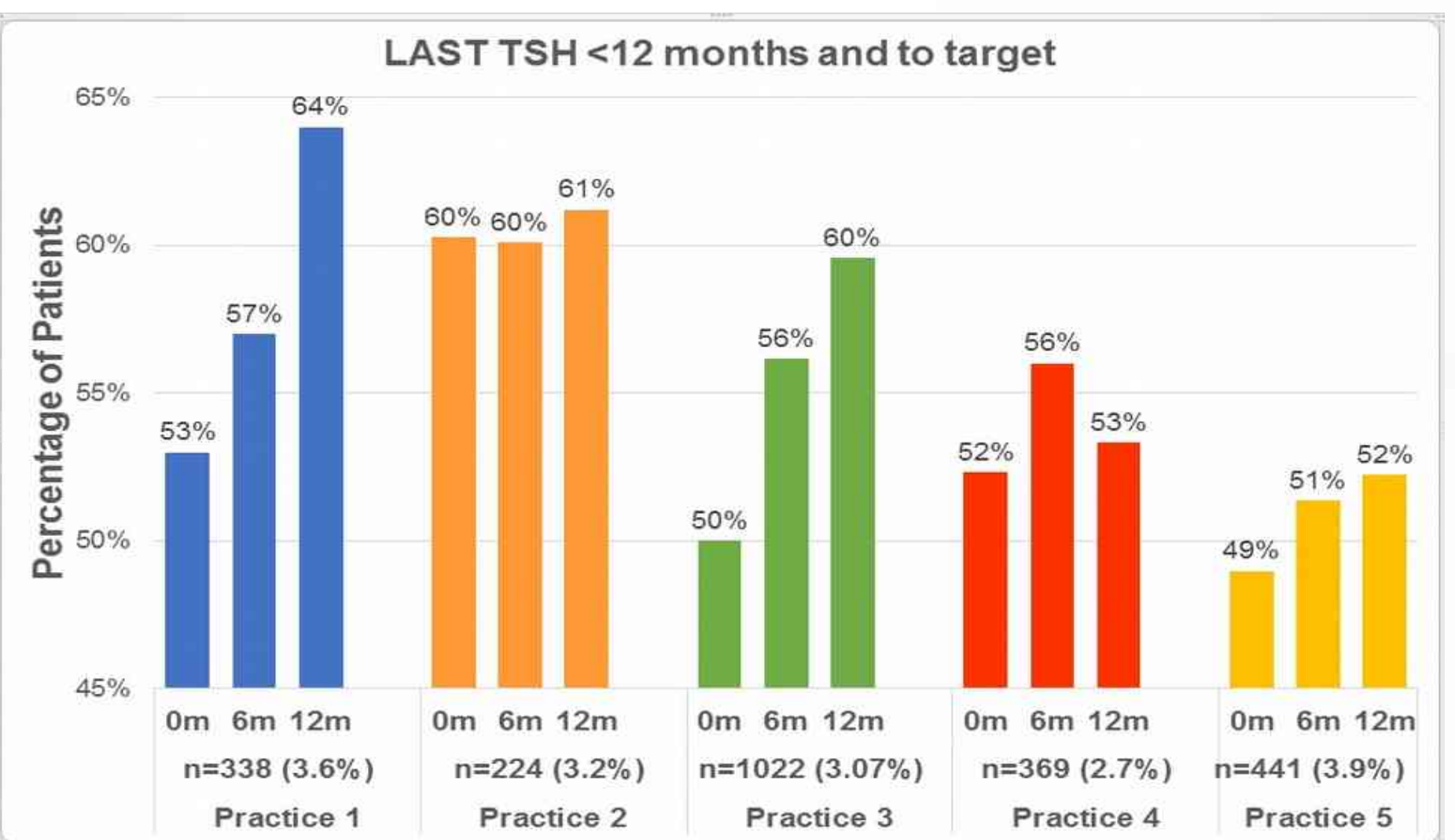

Table 2: Composite data from 5 practices

\begin{tabular}{|l|c|c|c|}
\hline PRACTICE & \multicolumn{3}{|c|}{ ALL PRACTICES (5) } \\
\hline PERIOD & Baseline & $6 \mathrm{~m}$ & $\mathbf{1 2 m}$ \\
\hline TOTAL & 74994 & 75387 & 75371 \\
POPULATION & $(7069-33358)$ & $(6931.33358)$ & $(6800-33386)$ \\
\hline TREATED PRIM & 2418 & 2399 & 2447 \\
HYPOTHYROIDISM & $(224-1022)$ & $(228-1015)$ & $(232-1036)$ \\
\hline TSH checked & $77 \%$ & $81 \%$ & $83 \%$ \\
In last 12m & $(74-81 \%)$ & $(78-83 \%)$ & $(80-85 \%)$ \\
\hline Last TSH 0.35-5 & $68 \%$ & $70 \%$ & $71 \%$ \\
(anv date) & $(66-73 \%)$ & $(68-72 \%)$ & $(66-75 \%)$ \\
\hline LAST TSH <12m & $53 \%$ & $56 \%$ & $58 \%$ \\
and TO TARGET & {$[49-60 \%]$} & {$[51-60 \%]$} & $52-64 \%]$ \\
\hline \hline \multicolumn{4}{|r}{} \\
\hline
\end{tabular}

\section{Conclusion}

- An electronic protocol which reminds GPs to check thyroid function and alerts them to TSH values that are out of range, resulted in modest improvements in monitoring and biochemical control of primary hypothyroidism

- Although both may improve over subsequent years, additional measures are likely to be required to achieve higher levels of monitoring and improvements in thyroid hormone replacement.

\section{References}

1. HSCIC Quality \& Outcome Framework GP Practice results

(http://www.qof.hscic.gov.uk/search/)

2. Minchin M et al. 2018. Quality of care in the United Kingdom after removal of financial incentives. N Engl J Med 2018; 379:948-957

3. Jepson L. Audit of the treatment of primary hypothyroidism in a general practice population. Endocrine Abstracts (2009) 19 P359

4. Tran A, et al. An electronic protocol replicating QOF thyroid alerts improves monitoring but does not help optimise levothyroxine replacement in hypothyroidism in primary care. Endocrine Abstracts 201750 P388.

5. Canaris GJ et al. The Colorado Thyroid Disease Prevalence Study. Arch Intern Med. 2000;160(4):526-534 